\section{New home for bromodomains}

\section{By Lauren Martz, Staff Writer}

Researchers at the Dana-Farber Cancer Institute and the Baylor College of Medicine have shown that Tensha Therapeutics Inc.'s bromodomain inhibitors could prevent sperm production and be developed as a male contraceptive. ${ }^{1}$ The biotech, which is focused on developing the molecules for cancer, has exclusive rights to the findings and is interested in pursuing bromodomain inhibitors in the indication.

Available options for male contraception are limited to barrier methods and vasectomy. There are no male contraceptive drugs on the market. Several hormone-based strategies are in the clinic but carry the risk of systemic side effects.

Indeed, Michael O'Rand, professor of cell biology and developmental biology at The University of North Carolina at Chapel Hill, said not all men react the same way to testosterone-based contraceptives and that testosterone can cause increased aggression.

"Prior genetic and our
chemical genetic studies
suggest that selective BRDT
inhibition will confer profound
effects on spermatogenesis
and fertility without
significant symptoms, in
particular without affecting
the male sex hormone axis."
-James Bradner,
Harvard Medical School

and staff physician in the Department of Medical Oncology at DanaFarber, and Martin Matzuk, professor of developmental biology at Baylor College of Medicine.

The paper also included researchers from the University of Oxford, Texas A\&M University, Boston Children's Hospital and The George Washington University School of Medicine and Health Sciences.

Pharmacologic and crystallography studies showed that JQ1 bound and competitively inhibited BRDT. In mice, intraperitoneal (i.p.) dosing resulted in significant concentrations of the compound in testicular tissue, suggesting the molecule is capable of crossing the blood-testis barrier and could be a good contraceptive candidate.

In mice, i.p. injection of JQ1 decreased testicular volume, seminiferous tube volume, number of spermatids, sperm count and sperm motility compared with vehicle injection. JQ1 did not affect serum levels of follicle stimulating hormone (FSH), luteinizing hormone ( $\mathrm{LH}$ ) or testosterone, suggesting that any contraceptive activity was caused by a hormoneindependent effect on the reproductive germ cells.

The team then tested the effect of the BRDT inhibitor on fertility and mating behavior in mice. Injection of $100 \mathrm{mg} / \mathrm{kg} /$ day of JQ1 completely prevented male mice from siring offspring when mated with females for one month. Lower doses reduced litter numbers and size, suggesting a dose-dependent contraceptive effect.

Finally, the team found that within one to three months of discontinuing JQ1, physiological effects of the drug including reduced testicular volume, sperm count and sperm motility were reversed. Fertility also was restored in the mice. Compared with vehicle-treated controls, mice previously treated with JQ1 sired similar size and number of litters and produced offspring of normal size, behavior and activity.

Results were published in Cell.

These issues suggest nonhormonal strategies could be more successful. The first clues that bromodomain inhibitors could be good candidates emerged in 2007 when a group at Columbia University Medical Center found that genetic knockout of bromodomain testisspecific (BRDT), a bromodomain-containing protein specifically found in the testis, caused sterility in male mice. ${ }^{2}$

Bromodomains are protein domains that bind to acetylated lysines and are involved in chromatin remodeling.

Additional evidence that BRDT could be a male contraceptive target came in 2010 when a group at The University of Utah School of Medicine showed that mutations in BRDT were associated with male infertility in a genomewide association study. ${ }^{3}$

Now, groups from Dana-Farber and Baylor have teamed up to determine how bromodomain inhibitors affect male fertility. The researchers found JQ1, a bromodomain inhibitor that acts against proteins including BRDT and bromodomain containing 4 (BRD4), had a hormone-independent contraceptive effect in male mice.

The groups were led by James Bradner, assistant professor in the Department of Medicine at Harvard Medical School and investigator

\section{Getting testes specific}

Bradner told SciBX that the next steps for pursuing the contraceptive indication include developing an inhibitor that is specific for BRDT.

Christina Wang, professor of medicine in the Division of Endocrinology \& Metabolism at the Los Angeles Biomedical Research Institute at Harbor-UCLA Medical Center, said special attention will need to be paid to the off-target effects of bromodomain inhibitors, which affect gene expression.

O'Rand said the team will need to prove that the compound does not affect mitosis elsewhere. In particular, he said activity on lymphocytes during an immune response should be analyzed because JQ1 originally was studied for its effect in cancers including myeloid leukemia.

Matzuk and Bradner believe that the design of BRDT-specific inhibitors should reduce the risks of these types of off-target effects. "Because JQ1 binds to some BRDT-related proteins, our goal is to develop a drug that blocks only BRDT and would theoretically have less side effects," Matzuk said. 
"Prior genetic and our chemical genetic studies suggest that selective BRDT inhibition will confer profound effects on spermatogenesis and fertility without significant symptoms, in particular without affecting the male sex hormone axis," said Bradner.

Wang said high regulatory standards and the proven safety and efficacy of female contraceptives may result in limited interest by pharmaceutical companies in BRDT inhibitors for male contraception.

O'Rand agreed that the FDA likely will set a higher bar for bromodomain inhibitors in contraception than it would in cancer but thinks the market is worth tackling.

"Female contraceptives are a huge industry estimated to be worth about $\$ 17$ billion by 2015 , and depending on how you collect the data, there are really good signs that male contraceptives could be accepted by a lot of people and that the market is really there," he said. "A lot of women are unable or unwilling to take the birth control pill for various reasons, so a real target is men in committed relationships with these women. Through surveys, we are also finding that men of certain demographics seem particularly responsive to the idea of taking a contraceptive pill."

Matzuk agreed that there is room for both male and female contraceptives. "The pill for women has the drawback that it alters hormone levels," he said.

Tensha CEO Doug Onsi said the company is interested in the potential of bromodomain inhibitors as male contraceptives but declined to provide a timeline for the program.

\section{Other angles}

Blocking spermatogenesis with strategies such as BRDT inhibition is not the only option for male contraception. For example, O'Rand said his team is developing a molecule that inhibits sperm motility to prevent fertilization of the egg.

He said, "A potential issue with a contraceptive that is shutting off the supply of sperm is that it will not be immediately effective. A patient would need to wait a long time, maybe more than 60 days, before the contraceptive activity kicks in. Like with a vasectomy, it might also be necessary to use some type of test with this contraceptive strategy to determine whether sperm production has stopped and when the treatment will be effective."

Wang agreed. She said blocking spermatogenesis may have slower activity by blocking sperm at the source than contraceptives that affect sperm after they have been produced.

Tensha's Onsi told SciBX that Dana-Farber filed patent applications covering the composition of matter for JQ1 and other bromodomain inhibitors. He said that Dana-Farber and Baylor jointly filed separate IP relating to use of the compounds in male contraception. Tensha has exclusive rights to both sets of IP.

Martz, L. SciBX 5(35); doi:10.1038/scibx.2012.914

Published online Sept. 6, 2012

\section{REFERENCES}

1. Matzuk, M.M. et al. Cell; published online Aug. 17, 2012; doi:10.1016/j.cell.2012.06.045

Contact: James E. Bradner, Dana-Farber Cancer Institute, Boston, Mass.

e-mail: james_bradner@dfci.harvard.edu

Contact: Martin M. Matzuk, Baylor College of Medicine, Houston, Texas

e-mail: mmatzuk@bcm.edu

2. Shang, E. et al. Development 134, 3507-3515 (2007)

3. Aston, K.I. et al. Hum. Reprod. 25, 1383-1397 (2010)

\section{COMPANIES AND INSTITUTIONS MENTIONED}

Baylor College of Medicine, Houston, Texas

Boston Children's Hospital, Boston, Mass.

Columbia University Medical Center, New York, N.Y.

Dana-Farber Cancer Institute, Boston, Mass.

The George Washington University School of Medicine and

Health Sciences, Washington, D.C.

Harbor-UCLA Medical Center, Torrance, Calif.

Harvard Medical School, Boston, Mass.

Los Angeles Biomedical Research Institute, Los Angeles, Calif.

Tensha Therapeutics Inc., Cambridge, Mass.

Texas A\&M University, College Station, Texas

The University of North Carolina at Chapel Hill, Chapel Hill, N.C. University of Oxford, Oxford, U.K.

The University of Utah School of Medicine, Salt Lake City, Utah 